\title{
UTILIZING LARGE HALL OFFSET VOLTAGE FOR CONVERSION FREE 4H-SIC STRAIN SENSOR
}

\author{
Tuan-Khoa Nguyen ${ }^{1}$, Hoang-Phuong Phan ${ }^{1}$, Jisheng Han ${ }^{1}$, Toan Dinh ${ }^{1}$, Abu Riduan Md Foisal ${ }^{1}$, \\ Yong Zhu ${ }^{1,2}$, Nam-Trung Nguyen ${ }^{l}$, and Dzung Viet Dao ${ }^{1,2}$ \\ ${ }^{1}$ Queensland Micro-Nanotechnology Centre, Griffith University, Brisbane, AUSTRALIA \\ ${ }^{2}$ School of Engineering, Griffith University, Gold Coast, AUSTRALIA
}

\begin{abstract}
This work presents a conversion free p-type $4 \mathrm{H}$ silicon carbide $(4 \mathrm{H}-\mathrm{SiC})$ four-terminal strain sensor utilizing a large Hall offset voltage in a symmetric fourterminal configuration. Upon the application of mechanical strain, a high sensitivity of $209 \mathrm{mV} / \mathrm{A} / \mathrm{ppm}$ was obtained. The strain sensor also exhibited good repeatability and linearity with a significantly large offset voltage in the induced strain ranging from 0 to $334 \mathrm{ppm}$. Coupled these performances with the excellent mechanical strength, electrical conductivity, thermal stability, and chemical inertness of the SiC material, the proposed $4 \mathrm{H}-\mathrm{SiC}$ strain sensor is promising for stress/strain monitoring for harsh operating environments with high signal-to-noise ratio.
\end{abstract}

\section{INTRODUCTION}

The piezoresistivity of materials can produce a large change of the resistivity upon the application of mechanical stresses/strains, thanks to the large piezoresistive effect in semiconductor materials. This phenomenon has been intensively utilized for mechanical sensors [1,2], such as strain gauges/sensors, pressure sensors, etc. In a vast number of mechanical sensing studies, the strain induced effect in two-terminal configurations was employed, where the resistance can linearly vary upon the application of external stresses/strains. The sensitivity of these devices is typically more significant when the electric current/voltage field is aligned parallel to the straining direction (i.e. longitudinal orientation). However, to measure the resistance variation in two-terminal configurations, external conversion circuitries are typically required to convert resistance to voltage (i.e. the Wheatstone bridge circuit). The difference in the temperature coefficient of the four resistors comprising the Wheatstone bridge circuit, leads to a non-zero offset during operations with varying temperature, hindering the sensitivity and reliability of the sensors [3-4]. The requirement for additional circuitries also obstructs the miniaturization and integration of sensing devices. These existing issues in two-terminal devices can be easily overcome by utilizing the anisotropic piezoresistance of four-terminal configurations in their crystal plane. As such, a significantly large voltage signal can be generated upon the application of mechanical stresses/strains without the need for conversion circuitries. These configurations are known as pseudo-Hall devices or Van der Pauw devices in the absence of an external magnetic field. In fact, a four-terminal silicon pressure sensor has been patented and commercialized since the 1980s [5]. Nevertheless, silicon-based devices face several drawbacks including the instability in harsh environments

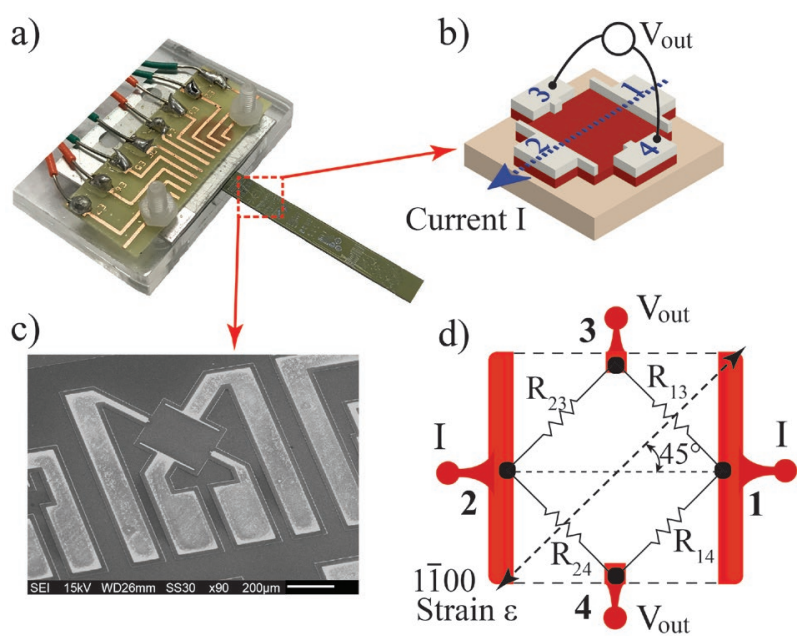

Figure 1: a) Optical image of experimental apparatus of the p-type 4H-SiC four-terminal strain sensor. b) Schema of the four-terminal configuration. c) SEM image of the sensor. d) Sensor alignment with applied strain in $<1 \overline{1} 00>$ direction.

(e.g. high temperature, high shock and chemically aggressive media) due to the low intrinsic energy bandgap of silicon. In contrast, silicon carbide ( $\mathrm{SiC})$ is of interest for high power electronics devices, and high temperature applications owing to its wide energy-band gap, high break-down voltage, thermal stability, and excellent chemical innerness [6]. Taking advantage of these superior properties and the availability of $\mathrm{SiC}$ wafers, many SiC-based MEMS mechanical sensors have been developed for harsh operating environments [7-10].

The piezoresistive effect, represented by the gauge factor (GF) [11] or piezoresistive coefficients of twoterminal $\mathrm{SiC}$ devices, has been utilized for mechanical sensing applications with relatively high GFs [12-19]. There are numerous studies on the $3 \mathrm{C}-\mathrm{SiC}$ and n-type $4 \mathrm{H}$ $\mathrm{SiC}$ based four-terminal strain sensors with a relatively high sensitivity and good reproducibility [4,20-22]. However, the strain induced effect and strain sensing in four-terminal p-type $4 \mathrm{H}-\mathrm{SiC}$ devices have not been investigated yet. This raises the need for understanding this phenomenon in such material to develop highly sensitive, circuit conversion free strain sensors.

This work demonstrates a highly sensitive $4 \mathrm{H}-\mathrm{SiC}$ four-terminal strain sensor, exhibiting good repeatability, linearity and high sensitivity. The use of a four-terminal structure eliminates the need for the resistance-to-voltage conversion, yielding a high sensitivity of $209 \mathrm{mV} / \mathrm{A} / \mathrm{ppm}$. The experimental results show that the sensitivity of the sensor was independent of the applied current. This is highly favorable for strain sensing applications where the calibration for the input current is no longer required. 
a)
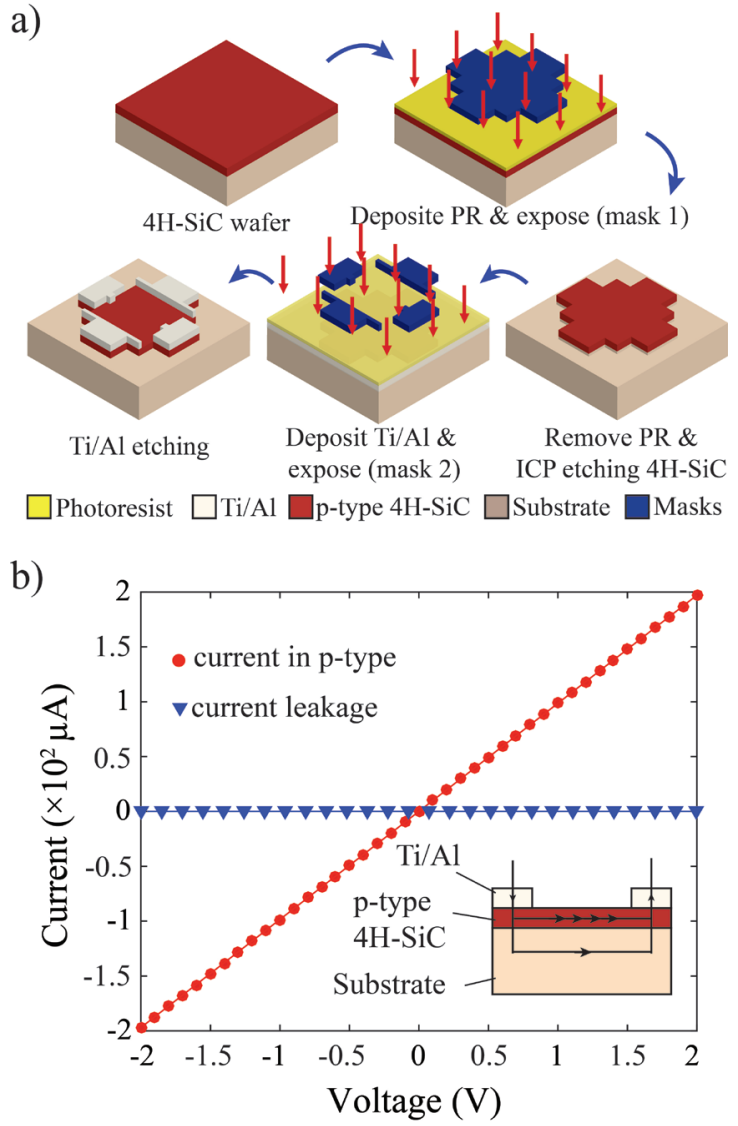

Figure 2: a) Device fabrication process flow. b) Linear current-voltage (I-V) characteristic of the contact to p-type 4H-SiC and current leakage to substrate. Inset: Schema of I-V measurement.

\section{CONFIGURATION OF 4H-SIC FOUR- TERMINAL STRAIN SENSOR}

The configuration of the $4 \mathrm{H}-\mathrm{SiC}$ four-terminal strain sensor is illustrated in Fig. 1, in which the current was supplied at the rectangular-shaped electrodes, while the output voltage was measured at the square-shaped electrodes. According to the square-type model for a fourterminal silicon-based pressure sensor [20-22], the sensor consists of four symmetric resistors $R_{13}, R_{23}, R_{24}$, and $R_{14}$ (Fig 1(d)). By supplying a constant current flowing between terminals 1 and 2 , in the stress-free state the circuit is in equilibrium (i.e. $V_{34}=V_{\text {offset }}=0$ ) due to $R_{13}=R_{23}=R_{24}=R_{14}=R_{0}$. Subsequently, by applying strain/stress, anisotropic variations of the four resistors would be obtained, depending on the orientation and magnitude of strain/stress applied to each resistor. Upon the application of uniaxial stress in the $<1 \overline{1} 00>$ orientation, there are asymmetric variations of the four piezoresistors, yielding an offset voltage between Hall terminals 3 and 4 (i.e. $V_{34}$ ). Consequently, the fourterminal configuration can be employed as a strain sensor where the initial induced strain can be monitored by measuring the output voltage $V_{\text {out }}=V_{34}$. The output signal $V_{\text {out }}$ is given by $[4,23]$ :

$$
V_{\text {out }}=\left(\frac{R_{24}}{R_{14}+R_{24}}-\frac{R_{23}}{R_{13}+R_{23}}\right) R_{12} I_{\text {in }}
$$

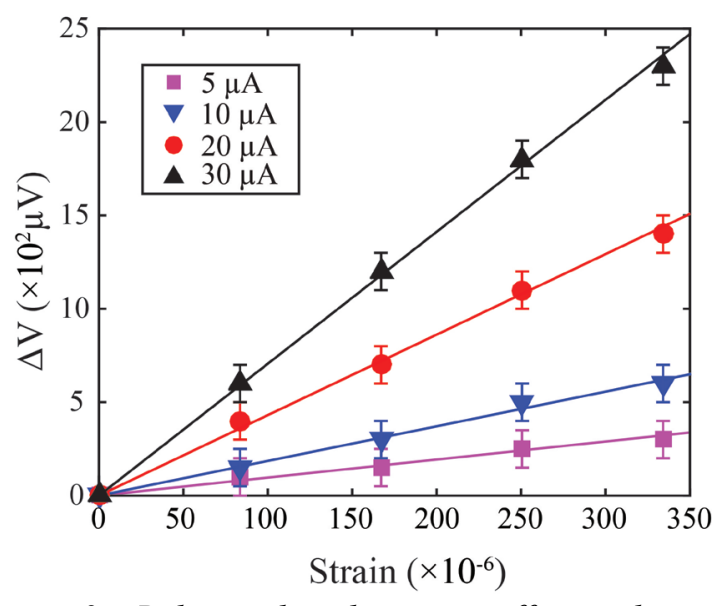

Figure 3: Relationship between offset voltage at terminals 3 and 4 of the strain sensor versus applied strains at various input currents from 5 to $30 \mu \mathrm{A}$.

where $I_{i n}$ and $R_{12}$ are the supplied current and resistance between terminals 1 and 2. Figure 3 shows a significant output voltage at different supplied currents from 5 to $30 \mu \mathrm{A}$. This is attributed to the fact that $R_{23}$ and $R_{14}$ were elongated in the longitudinal direction parallel to the flowing current whereas $R_{13}$ and $R_{24}$ were stretched in the transverse direction perpendicular to the current Therefore, $R_{23} / R_{14}$ and $R_{13} / R_{24}$ vary with tensile stress by the longitudinal GF (increase) and transverse GF (decrease), respectively.

\section{FABRICATION AND EXPERIMENTAL RESULT}

A 4H-SiC (0001) wafer with a thickness of $350 \mu \mathrm{m}$ was used to fabricate the four-terminal strain sensor, which consists of a $1 \mu \mathrm{m} 10^{18} \mathrm{~cm}^{-3}$ doped p-type epilayer as the functioning layer. A $1 \mu \mathrm{m}$ n-type buffer layer was deposited on the top of a low-doped n-type substrate. Figure 2(a) presents the fabrication of the p-type $4 \mathrm{H}-\mathrm{SiC}$ four-terminal strain sensor fabricated by conventional micro-machined processes. As such, a $4.3 \mu \mathrm{m}$-thick AZ9245 photoresist was spun coating for a subsequent inductive coupled plasma (ICP) etching of the p-type layer. The etching was carried out using a STS ${ }^{\mathrm{TM}}$ etcher with $\mathrm{HCl}$ at a pressure of 2 mTorr. The ICP etch rate was $100 \mathrm{~nm}$-per-minute and the etched depth of $1.3 \mu \mathrm{m}$ was achieved, ensuring that the $1 \mu \mathrm{m}$ p-type layer was thoroughly etched in the designated area. Consequently, mesa functioning structures were formed on the p-type layer. $100 \mathrm{~nm}$ titanium and aluminum layers were subsequently deposited for making the contact. Next, $\mathrm{Ti} / \mathrm{Al}$ was wet etched, followed by an annealing at high temperature up to $1000^{\circ} \mathrm{C}$. Finally, the wafer was diced into $30 \mathrm{~mm} \times 3 \mathrm{~mm}$ rectangular beams which were aligned to the $<1 \overline{1} 00>$ direction.

Figure 2(b) shows a good linearity of the currentvoltage characteristics of the annealed $\mathrm{Ti} / \mathrm{Al}$ contact to $\mathrm{p}$ type $4 \mathrm{H}-\mathrm{SiC}$ in the voltage range from -2 to $2 \mathrm{~V}$. Accordingly, the resistivity of the p-type layer was found to be $0.91 \Omega \mathrm{cm}$. Since strain sensors typically operate in relatively low voltage ranges (e.g. below $\pm 1 \mathrm{~V}$ ) to avoid 


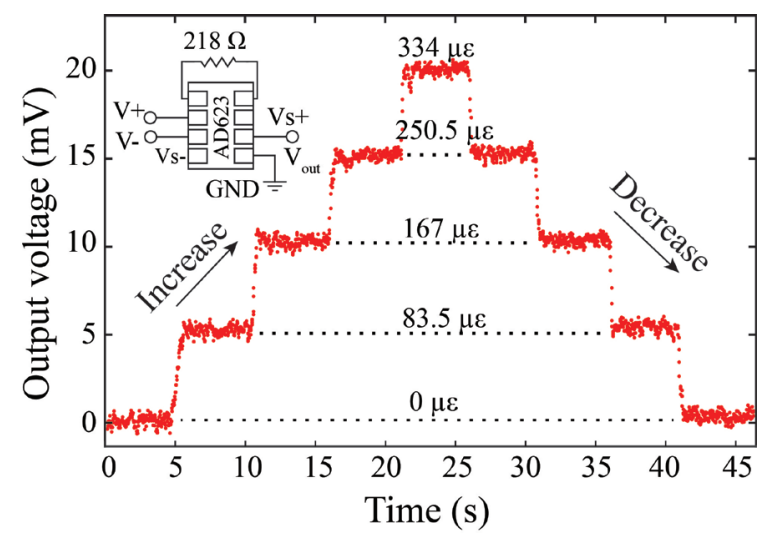

Figure 4: Real-time strain monitoring by the $4 \mathrm{H}$ SiC four-terminal strain sensor with stepped strains from 0 to 334ppm. The offset voltage was amplified with a gain $g$. Inset: Amplifying circuit configuration using AD623.

the Joule heating effect, the measured I-V characteristic in the given range is sufficient for strain sensing applications. When sweeping bias voltages from -2 to $2 \mathrm{~V}$, the leakage current to the n-type layer and the substrate is seven orders of magnitude smaller than the current in the sensor. This is attributed to the fact that the back-to-back $\mathrm{p}-\mathrm{n}$ diode prevents the current from leaking into the ntype layer and the substrate $[17,18]$, ensuring that only the p-type layer contributes to the measurement.

In the present work, the operation of the $4 \mathrm{H}-\mathrm{SiC}$ fourterminal strain sensor under uniaxial strain/stress was performed using a bending beam method. This technique was widely employed for the characterizations of the strain induced effect in semiconductors [9,12,16-18]. As can be seen in Fig. 1(a), the SiC beam with one tightly clamped end was bent downward by applying a static force at the free end. Since the beam has a cantilevershape, the bending would yield a uniaxial strain to the top p-type layer, which is parallel to the longer edges of the beam. This straining configuration has been reported elsewhere $[9,12,17]$, in which uniaxial strain is effectively transferred to the mesa top structures: $\varepsilon=6 F l / E_{S i C} b t^{2}$, where $F$ is the applied force; $l, b, t$ and $E_{\mathrm{SiC}}$ are the length, width, thickness and Young's modulus of the 4H-SiC beam, respectively. The stress induced can be deduced from the Hooke's law: $\sigma=\varepsilon E_{\mathrm{SiC}}$.

The output voltage linearly varied with the applied strain ranging from 0 to $334 \mathrm{ppm}$ (equivalent to an induced stress from 0 to $168 \mathrm{MPa}$ ). This indicates the negligible Joule heating effect and the current leakage to the n-type layer and the substrate. In the bending experiment, a uniaxial stress was applied in the $<1100>$ orientation parallel to the diagonal axis of the sensor. As such, $R_{23}$ and $R_{14}$ were elongated in the longitudinal direction parallel to the flowing current while $R_{13}$ and $R_{24}$ were stretched in the transverse direction perpendicular to the current. The relationship of the four resistors upon strain is given by:

$$
\left\{\begin{array}{l}
R_{23}=R_{14}=R_{0}\left(1+\Gamma_{L} \varepsilon\right) \\
R_{13}=R_{24}=R_{0}\left(1+\Gamma_{T} \varepsilon\right)
\end{array}\right.
$$

where $\Gamma_{\mathrm{L}}$ and $\Gamma_{\mathrm{T}}$ are longitudinal and transverse gauge factors of p-type $4 \mathrm{H}-\mathrm{SiC}$, which have been reported elsewhere [17]. Consequently, the sensitivity $S$ of the $4 \mathrm{H}-$ $\mathrm{SiC}$ four-terminal strain sensor is given by:

$$
S=\frac{V_{\text {out }}}{\varepsilon I_{\text {in }}}
$$

From the experimental result, the sensitivity of the sensor was measured as high as $209 \mathrm{mV} / \mathrm{A} / \mathrm{ppm}$, which is approximately three times larger than a reported result of 3C-SiC sensor [20]. Figure 3 shows the relationship between the output voltage $V_{\text {out }}$ versus the induced strain up to $334 \mathrm{ppm}$. Evidently, the linear response of the sensor's signal was obtained with different applied currents ranging from 5 to $30 \mu \mathrm{A}$. At a certain strain, the output voltage of the sensor was proportional to the applied current. In other words, the sensitivity of the sensor is independent of the applied current. This characteristic is highly favorable for strain sensing, eliminating the sensor's calibration for different input currents.

Figure 4 shows a demonstration of the real-time strain monitoring using the $4 \mathrm{H}-\mathrm{SiC}$ four-terminal strain sensor. Stepped strains were continuously applied from 0 to $334 \mathrm{ppm}$ at the input current of $20 \mu \mathrm{A}$. The output signal was amplified and monitored using an amplifying circuit (Fig. 4: Inset). The gain $g$ of the amplifying circuit can be adjustable to obtain the desired output signal. Under stepped strain, the sensor exhibited linear response in the strain range from 0 to $334 \mathrm{ppm}$. When the load was completely removed, the output signal returned to its initial value. Additionally, the output was extremely stable at each straining level without any signal drift after hundreds of loading cycles. The measured results indicate a good linearity and repeatability of the $4 \mathrm{H}-\mathrm{SiC}$ fourterminal strain sensor. These characteristics are of interest for the development of highly sensitive and high signalto-noise ratio mechanical strain sensors.

\section{CONCLUSION}

A highly sensitive $4 \mathrm{H}-\mathrm{SiC}$ four-terminal strain sensor was presented using a four-terminal configuration which simplifies the read-out circuit without the need for the Wheatstone bridge to convert resistance change to voltage. By utilizing a bending beam method to apply uniaxial strain/stress to the sensor, good repeatability and linearity were obtained as well as a high sensitivity of 209 $\mathrm{mV} / \mathrm{A} / \mathrm{ppm}$. It was observed the sensitivity of the sensor was independent of the applied current. The as-developed $4 \mathrm{H}-\mathrm{SiC}$ four-terminal strain sensor is promising for highly sensitive and low noise mechanical sensing in high temperature operations.

\section{ACKNOWLEDGEMENTS}

This work has been partially supported by Australian Research Council grants LP150100153 and LP160101553. This work was also supported by the Queensland node of the Australian National Fabrication Facility, a company established under the National Collaborative Research Infrastructure Strategy to provide nano and micro-fabrication facilities for Australia's researchers. 


\section{REFERENCES}

[1] T.-V. Nguyen, T. Tsukagoshi, H. Takahashi, K. Matsumoto, and I. Shimoyama, "Depinning-induced capillary wave during the sliding of a droplet on a textured surface", Langmuir, vol. 32(37), pp. 9523 9529, 2016

[2] T.-V. Nguyen, M.-D. Nguyen, H. Takahashi, K. Matsumoto, and I. Shimoyama, "Viscosity measurement based on the tapping-induced free vibration of sessile droplets using MEMS-based piezoresistive cantilevers", Lab Chip, vol. 15, pp. 3670-3676, 2015.

[3] A. Mian, J. C. Suhling, and R. Jaeger, "The Van der Pauw stress sensor", IEEE Sens. J., vol. 6, pp. 340 356, 2006.

[4] A. Qamar, H.-P. Phan, J. Han, P. Tanner, T. Dinh, L. Wang, D. V. Dao and S. Dimitrijev, "The effect of device geometry and crystal orientation on the stressdependent offset voltage of $3 \mathrm{C}-\mathrm{SiC}(100)$ four terminal devices", J. Mater. Chem. C, vol. 34(3), pp. 8804-8809, 2015

[5] Motorola, Inc, "Silicon pressure sensor", US patent US4317126A, 1982

[6] H.-P. Phan, I. Ginosuke, T. Dinh, T. Kozeki, T.-K. Nguyen, T. Namazu, A. Qamar, D. V. Dao, and N.-T. Nguyen, "Formation of silicon carbide nanowire on insulator through direct wet oxidation", Mater. Lett., vol. 196, pp. 280-283, 2017.

[7] D. G. Senesky, B. Jamshidi, K. B. Cheng, and A. Pisano, "Harsh Environment Silicon Carbide Sensors for Health and Performance Monitoring of Aerospace Systems: A Review”, IEEE Sensors J., vol. 9, no. 11, pp. 1472-1478, 2009

[8] H.-P. Phan, H.H. Cheng, T. Dinh, B. Wood, T.-K. Nguyen, F. Mu, H. Kamble, R. Vadivelu, G. Walker, L. Hold, A. Iacopi, B. Haylock, D.V. Dao, M. Lobino, T. Suga, and N.-T. Nguyen, "SingleCrystalline 3C-SiC anodically Bonded onto Glass: An Excellent Platform for High-Temperature Electronics and Bioapplications" ACS Appl. Mater. Interfaces., vol. 9, pp. 27365-27371, 2017.

[9] H.-P. Phan, T. Dinh, T. Kozeki, A. Qamar, T. Namazu, S. Dimitrijev, N.-T. Nguyen, and D. V. Dao, "Piezoresistive effect in p-type $3 \mathrm{C}$-SiC at high temperatures characterized using Joule heating", Sci. Rep., vol. 6, pp. 28499, 2016.

[10] T. Akiyama, D. Briand, and N.F.de Rooij, "Designdependent gauge factors of highly doped n-type $4 \mathrm{H}-$ SiC piezoresistors", in Proc. IEEE Sensors, Ireland, October 28-31, 2011, pp. 222-225.

[11] T.-K. Nguyen, T. Dinh, H.-P. Phan, C.-D. Tran, A. R. Md Foisal, Y. Zhu, and D. V. Dao, "Electrically stable carbon nanotube yarn under tensile strain", IEEE Electron Device Lett., vol. 38(9), pp. 13311334, 2017.

[12]H.-P. Phan, T. Dinh, T. Kozeki, T.-K. Nguyen, A. Qamar, T. Namazu, N.- T. Nguyen, and D. V. Dao, "Nano strain-amplifier: Making ultra-sensitive piezoresistance in nanowires possible without the need of quantum and surface charge effects", Appl. Phys. Lett., vol. 109, no. 12, pp. 123502, 2016.

[13] J. Bi, G. Wei, L. Wang, F. Gao, J. Zheng, B. Tang, and W. Yang, "Highly sensitive piezoresistance behaviors of n-type 3C-SiC nanowires", J. Mater. Chem. C, vol. 1, no. 30, pp. 4514-4517, 2013.

[14]F. Gao, J. Zheng, M. Wang, G. Wei, and W. Yang, "Piezoresistance behaviors of p-type 6H-SiC nanowires", Chem. Commun., vol. 47, pp. $11993-$ 11995, 2011.

[15]X. Li, S. Chen, P. Ying, F. Gao, Q. Liu, M. Shang, and W. Yang, "A giant negative piezoresistance effect in 3C-SiC nanowires with B dopants", $J$. Mater. Chem. C, vol. 4, pp. 6466-6472, 2016.

[16] H.-P. Phan, T.-K. Nguyen, T. Dinh; G. Ina, A. R. Kermany, A. Qamar, J. Han, T. Namazu, R. Maeda, D.V. Dao, N.-T. Nguyen, "Ultra-high strain in epitaxial silicon carbide nanostructures utilizing residual stress amplification", Appl. Phys. Lett., vol. 110, pp. 141906, 2017

[17] T.-K. Nguyen, H.-P. Phan, T. Dinh, J. Han, S. Dimitrijev, P. Tanner, A. R. Md Foisal, Y. Zhu, N. T. Nguyen and D. V. Dao, "Experimental investigation of piezoresistive effect in p-type 4H-SiC", IEEE Electron Device Lett., vol. 38(7), pp. 955-958, 2017.

[18] T. Akiyama, D. Briand, and N. F. de Rooij, "Designdependent gauge factors of highly doped n-type $4 \mathrm{H}-$ SiC piezoresistors", J. Micromech. Microeng., vol. 22. no. 8, pp. 085034, 2012.

[19] R. S. Okojie, A. A. Ned, A. D. Kurtz, and W. N. Carr, "Characterization of highly doped n- and p-type 6H-SiC piezoresistors", IEEE Trans. Electron Devices, vol. 45, no. 4, pp. 785-790, 1998.

[20] A. Qamar, H.-P. Phan, D. Dao, P. Tanner, T. Dinh, L. Wang, and S. Dimitrijev, "The dependence of offset voltage in p-type $3 \mathrm{C}$-SiC van der Pauw device on applied strain", IEEE Electron Device Lett., vol. 36, no. 7, pp. $708-710,2015$.

[21]H. P. Phan, A. Qamar, D. V. Dao, T. Dinh, L. Wang, J. Han and N. T. Nguyen, "Orientation dependence of the pseudo-Hall effect in p-type $3 \mathrm{C}-\mathrm{SiC}$ fourterminal devices under mechanical stress", RSC $A d v$., vol. 5, pp. 56377-56381, 2015.

[22] Y. Kanda and K. Yamamura, "Four-terminal-gauge quasi-circular and square diaphragm silicon pressure sensors", Sens. Actuators, vol. 18, no. 3, pp. 247257, 1989.

[23] Y. Kanda and A. Yasukawa, "Hall-effect devices as strain and pressure sensors", Sens. Actuators, vol. 2, no. 3, pp. 283-296, 1982.

\section{CONTACT}

*T-. K. Nguyen, tel: +61-424-351984;

khoa.nguyentuan@griffithuni.edu.au 